\title{
EXTREMAL FUNCTIONS WITH VANISHING CONDITION
}

\author{
FRIEDRICH LITTMANN AND MARK SPANIER
}

\begin{abstract}
For a Hermite-Biehler function $E$ of mean type $\tau$ we determine the optimal (with respect to the de Branges measure of $E$ ) majorant $M_{E}^{+}$and minorant $M_{E}^{-}$of exponential type $\tau$ for the truncation of $x \mapsto\left(x^{2}+a^{2}\right)^{-1}$. We prove that

$$
\int_{\mathbb{R}}\left(M_{E}^{+}(x)-M_{E}^{-}(x)\right)|E(x)|^{-2} d x=\frac{1}{a^{2} K(0,0)}
$$

where $K$ is the reproducing kernel for the de Branges space $\mathcal{H}(E)$. As an application we determine the optimal majorant and minorant for the Heaviside function that vanish at a fixed point $\alpha=i a$ on the imaginary axis. We show that the difference of majorant and minorant has integral value $(\pi a-$ $\tanh (\pi a))^{-1} \pi a$.
\end{abstract}

\section{Results}

Let $\delta \geq 0$. We say that an entire function $F$ is of exponential type $\delta \geq 0$ if for every $\varepsilon>0$ there exists $C_{\varepsilon}>0$ such that $|F(z)| \leq C_{\varepsilon} e^{(\delta+\varepsilon)|z|}$ for all $z \in \mathbb{C}$, and we write $\mathcal{A}(\delta)$ for the class of entire functions of exponential type $\delta$.

Let $\mu$ be a Borel measure on $\mathbb{R}$. We write $\mathcal{A}_{p}(\delta, \mu)$ for the space of functions $F \in \mathcal{A}(\delta)$ such that

$$
\int_{\mathbb{R}}|F(x)|^{p} d \mu(x)<\infty
$$

and we write $\mathcal{A}_{p}(\delta)$ if $\mu$ is Lebesgue measure.

Let $f: \mathbb{R} \rightarrow \mathbb{R}$ be measurable and of polynomial growth, and let $\alpha \in \mathbb{C} \backslash \mathbb{R}$. Consider the class $\mathcal{F}_{+}=\mathcal{F}_{+}(f, \alpha) \subseteq \mathcal{A}(\delta)$ of entire functions $F$ satisfying

(i) $F(x) \geq f(x)$ for all real $x$,

(ii) $F(\alpha)=0$,

(iii) $\|F-f\|_{L^{1}(\mathbb{R})}<\infty$.

Assume that $\mathcal{F}_{+} \neq \emptyset$. We seek to find $F^{+} \in \mathcal{F}_{+}$such that the inequality

$$
\int_{\mathbb{R}}\left(F(x)-F^{+}(x)\right) d x \geq 0
$$

holds for all $F \in \mathcal{F}_{+}$. If $F^{+}$exists, we call it an extremal majorant of $f$ (of type $\delta)$ with vanishing at $\alpha$. The class $\mathcal{F}_{-}(f, \alpha)$ is defined analogously by reversing the inequalities in (i) and (1.1); the corresponding optimal functions are called extremal minorants with vanishing at $\alpha$. We note that condition (ii) implies $F(\bar{\alpha})=0$.

Date: March 23, 2022.

2000 Mathematics Subject Classification. Primary 41A30, 41A52. Secondary 41A05, 41A44, $42 \mathrm{~A} 82$.

Key words and phrases. Exponential type, bandlimited functions, best one-sided approximation, de Branges space, Hermite-Biehler entire function. 
This extremal problem was first considered in 14 for the function

$$
f(x)=\chi_{[\beta, \gamma]}(x)
$$

with $\beta<\gamma$. M. Kelly found non-optimal functions in $\mathcal{F}_{+}\left(\chi_{[\beta, \gamma]}, \alpha\right)$ and obtained bounds for $\left\|F^{ \pm}-\chi_{[\beta, \gamma]}\right\|_{1}$ as a function of $\delta$. This choice of $f$ is quite natural, since the extremal functions satisfying (i) and (1.1) have found frequent applications in analytic number theory [10, 23, 26] and signal processing [9].

The condition (ii) may allow applications to certain explicit formulas for $L$ functions. We briefly describe the setup. An $L$-function $s \mapsto L(\chi, s)$ is given by

$$
L(\chi, s)=\sum_{n=1}^{\infty} \frac{\chi(n)}{n^{s}}
$$

where $\chi$ is a Dirichlet character. The generalized Riemann hypothesis states that the zeros of $L$ in $0<\Re s<1$ all lie on the line $\Re s=1 / 2$. An explicit formula is an identity that relates a series involving values $L(\chi, \rho) h(\rho)$ where $L(\chi, \rho)=0$ and $h$ is a smooth function to values of the Fourier transform of $h$. Under assumption of GRH and writing $f(t)=L\left(\chi, \frac{1}{2}+i t\right)$ the series involves only real values $t$, and $h$ is frequently chosen to be a solution to the above extremal problem. Without assumption of GRH the series will involve non-real values of $t$, and an extremal function satisfying (ii) may be used to eliminate the contribution of such a zero.

The results in this paper are an attempt to understand how the vanishing condition affects the construction of extremal functions. For this purpose we consider $\alpha=i a$ with $a>0$ and approximate the cutoff function $x_{+}^{0}$ defined by

$$
x_{+}^{0}=\left\{\begin{array}{l}
1 \text { if } x \geq 0 \\
0 \text { else }
\end{array}\right.
$$

We find the extremal majorant and minorant $F^{ \pm} \in \mathcal{F}_{ \pm}\left(x_{+}^{0}, i a\right)$ of type $2 \pi$. The results, given in Theorem 2 below, are obtained by changing measure and function in such a way that condition (ii) may be dropped. This is possible since $F^{+}$and $F^{-}$are necessarily of the form

$$
F^{ \pm}(z)=G^{ \pm}(z)\left(z^{2}+a^{2}\right)
$$

for some entire functions $G^{ \pm} \in \mathcal{A}(\delta)$. Hence, defining $t_{a}$ by

$$
t_{a}(x)=\left\{\begin{array}{l}
\left(x^{2}+a^{2}\right)^{-1} \text { if } x \geq 0, \\
0 \text { else }
\end{array}\right.
$$

we seek to find $G^{+}, G^{-} \in \mathcal{A}(\delta)$ such that $G^{-}(x) \leq t_{a}(x) \leq G^{+}(x)$ for all real $x$, and the integral

$$
\int_{-\infty}^{\infty}\left(G^{+}(x)-G^{-}(x)\right)\left(x^{2}+a^{2}\right) d x
$$

is minimal. This type of problem has been frequently investigated. An early result is the solution by Beurling [2] and independently by Selberg [23, Chapter 20] for the signum function, cf. the account in Vaaler [26]. Further references to recent developments can be found in [8]. For the related subject of one-sided polynomial approximation to the signum function see Bustamante et.al. [3], while the best one-sided approximations to characteristic functions by trigonometric polynomials were found recently by Babenko et.al. 11. For the best approximation without constraints we refer to Ganzburg [1]. For weighted polynomial approximation see 
Lubinsky [19, 20. For general facts regarding best approximation we refer to the books of Korneichuk [16] and Pinkus [21.

In Theorem 1 we solve the extremal problem for $t_{a}$ with respect to a large class of measures. In order to state this result we require some terminology from the theory of de Branges Hilbert spaces of entire functions. We refer to 4 and [13] for proofs of the following facts.

For any entire function $E$ we use the notation $E^{*}(z)=\overline{E(\bar{z})}$. An entire function $E$ satisfying the inequality

$$
|E(z)|>\left|E^{*}(z)\right|
$$

for all $z$ with $\Im z>0$ will be called a Hermite-Biehler (HB) function. An analytic function $F$ defined in the upper half plane $\mathbb{C}^{+}$is said to have bounded type, if $F$ is the quotient of two bounded functions. The number $v(F)$ defined by

$$
v(F)=\limsup _{y \rightarrow \infty} y^{-1} \log |F(i y)|
$$

is called the mean type of $F$. If $F$ has bounded type in $\mathbb{C}^{+}$, then its mean type is finite.

We denote by $\mathcal{H}(E)$ the vector space of entire functions $F$ such that

$$
\int_{-\infty}^{\infty}|F(x) / E(x)|^{2} d x<\infty
$$

and the functions $F / E$ and $F^{*} / E$ have bounded type and nonpositive mean type in $\mathbb{C}^{+}$. It is a Hilbert space with scalar product

$$
\langle F, G\rangle_{E}=\int_{-\infty}^{\infty} F(x) G^{*}(x)|E(x)|^{-2} d x .
$$

We define $A=(1 / 2)\left(E+E^{*}\right)$ and $B=(i / 2)\left(E-E^{*}\right)$. A fundamental result of de Branges from the 1960's is the recognition that this space is a reproducing kernel Hilbert space; we briefly sketch the argument. It follows from (1.2) that $E$ has no zeros in the open upper half plane, and it follows from the definition of $\mathcal{H}(E)$ that $F / E$ and $F^{*} / E$ have no zeros in an open set containing the closed upper half plane. The condition that $F / E$ and $F^{*} / E$ have non-positive mean type implies that the Cauchy integral formula for the upper half plane holds for $F / E$ and $F^{*} / E$ (e.g., 4. Theorem 12], note also that Cauchy formulas for $F / E^{*}$ and $F^{*} / E^{*}$ in the lower half plane hold), and it follows with an elementary calculation that $\mathcal{H}(E)$ has the reproducing kernel $K$ given by

$$
K(w, z)=\frac{B(z) A(\bar{w})-A(z) B(\bar{w})}{\pi(z-\bar{w})}
$$

for $z \neq \bar{w}$.

The prototypical example of a de Branges space is the classical Paley-Wiener space of square integrable functions of finite exponential type at most $\tau$. In this case $E(z)=e^{-i \tau z}$, and the condition that $F / E$ and $F^{*} / E$ have non-positive mean type implies that $F$ and $F^{*}$ grow no faster than $E$, i.e., this condition is essentially equivalent to the statement that $F$ has exponential type $\tau$. The reproducing kernel for this space is the familiar sinc function

$$
(z, w) \mapsto \frac{\sin \tau(z-\bar{w})}{\pi(z-\bar{w})} .
$$


The classical proof that this is a reproducing kernel uses Fourier analysis, but this can also be shown using a contour integration argument. It is the latter proof that generalizes to the setting of de Branges spaces.

We require the following conditions for $E$ :

(I) $E$ is of bounded type in $\mathbb{C}^{+}$with mean type $\tau$,

(II) $E$ has no real zeros,

(III) $E^{*}(z)=E(-z)$ for all $z$,

(IV) $B \notin \mathcal{H}(E)$.

These are technical assumptions; we return to these properties in Section 3 . where we obtain the following result.

Theorem 1. Let $E$ be a HB-function satisfying conditions (I) - (IV). If $F^{+}, F^{-} \in$ $\mathcal{A}(2 \tau)$ with

$$
F^{-}(x) \leq t_{a}(x) \leq F^{+}(x)
$$

for all real $x$, then

$$
\int_{\mathbb{R}}\left(F^{+}(x)-F^{-}(x)\right) \frac{d x}{|E(x)|^{2}} \geq \frac{1}{a^{2} K(0,0)},
$$

and there exist functions $T_{a}^{ \pm} \in \mathcal{A}(2 \tau)$ satisfying (1.4) such that there is equality in (1.5) for $F^{+}=T_{a}^{+}$and $F^{-}=T_{a}^{-}$.

We consider now the problem of finding the extremal functions with vanishing at $\alpha=i a$ with respect to Lebesgue measure. In order to apply Theorem 1 we require a Hermite-Biehler function $E_{a}$ satisfying (I) - (IV) such that

$$
\int_{-\infty}^{\infty}\left|\frac{F(x)}{E_{a}(x)}\right|^{2} d x=\int_{-\infty}^{\infty}|F(x)|^{2}\left(x^{2}+a^{2}\right) d x
$$

for all $F \in \mathcal{A}_{2}\left(\pi,\left(x^{2}+a^{2}\right) d x\right)$. We shall prove in Theorem 13 that $E_{a}$ defined by

$$
E_{a}(z)=\left(\frac{2}{\sinh (2 \pi a)}\right)^{\frac{1}{2}} \frac{\sin \pi(z+i a)}{z+i a} .
$$

has this property. Theorem 1 becomes applicable and we obtain the following result for extremal functions with vanishing at $\alpha=i a$.

Theorem 2. Let $a>0$. If $S, T \in \mathcal{A}(2 \pi)$ with

$$
S(i a)=T(i a)=0
$$

and

$$
S(x) \leq x_{+}^{0} \leq T(x)
$$

for all real $x$, then

$$
\int_{-\infty}^{\infty}(S(x)-T(x)) d x \geq \frac{\pi a}{\pi a-\tanh (\pi a)},
$$

and there exist $S_{a}^{+}, S_{a}^{-} \in \mathcal{A}(2 \pi)$ such that there is equality in (1.7) for $S=S_{a}^{-}$and $T=S_{a}^{+}$. 
It is instructive to consider the corresponding integral for the extremal functions of type $2 \pi \delta$. To find these values, we temporarily set $a=b \delta$. Then the extremal functions $F^{ \pm} \in \mathcal{A}(2 \pi)$ with $F(i b \delta)=0$ satisfy

$$
\int_{-\infty}^{\infty}\left(F^{+}(x)-F^{-}(x)\right) d x=\frac{\pi b \delta}{\pi b \delta-\tanh (\pi b \delta)} .
$$

We note that the functions $F_{\delta}^{ \pm}$given by $F_{\delta}^{ \pm}(z)=F^{ \pm}(\delta z)$ are the extremal functions of type $2 \pi \delta$ with $F_{\delta}^{ \pm}(i b)=0$, and a change of variable gives

$$
\int_{-\infty}^{\infty}\left(F_{\delta}^{+}(x)-F_{\delta}^{-}(x)\right) d x=\frac{\pi b}{\pi b \delta-\tanh (\pi b \delta)} .
$$

This implies that for fixed $b$ and $\delta \rightarrow \infty$ the integral is $\sim \delta^{-1}$, while for $\delta \rightarrow$ $0+$ the integral is $\sim 3(\pi b)^{-2} \delta^{-3}$. In [26, Theorem 8] the corresponding extremal problem for the signum function without the vanishing condition is treated. The integral value is shown to be equal to $\delta^{-1}$. This shows that the prescribed vanishing at $\alpha=i b$ substantially affects the integral value for small values of $\delta$, but the vanishing condition leads only to a small change if $\delta$ becomes large.

This paper is structured as follows. In Section 2 we give a general method to construct entire functions $M^{ \pm}$that interpolate $t_{a}$ at the zeros of a given LaguerrePólya entire function $F$, and we prove that

$$
x \mapsto F(x)\left(M^{ \pm}(x)-t_{a}(x)\right)
$$

is of one sign for all real $x$. These functions serve as candidates for the extremal functions in the approximation results of the subsequent sections. We prove Theorem 1 in Section 3 . In Section 4 we investigate the Hermite-Biehler function $E_{a}$ from (1.6) and prove Theorem 2

Acknowledgement. The authors thank the anonymous referees for their valuable suggestions.

\section{INTERPOLATION AT ZEROS OF LP-FUNCTIONS}

For certain discrete subsets $\mathcal{T} \subset \mathbb{R}$ we show how to construct entire functions $F \in \mathcal{A}(2 \tau)$ which interpolate $t_{a}$ at the elements of $\mathcal{T}$ (with prescribed multiplicity) so that $F-t_{a}$ has no sign changes between consecutive elements of $\mathcal{T}$. This is the basis for the construction of the extremal functions in Theorems 1 and 2 ,

The interpolation theorems of this section are based on the representation

$$
\frac{a}{z^{2}+a^{2}}=\int_{0}^{\infty} e^{-z \lambda} \sin (a \lambda) d \lambda
$$

valid for all $z$ with $\Re z>0$. The analogues of the crucial inequalities in Theorems 9 and 10 below for the function $x \mapsto e^{-x \lambda} \chi_{[0, \infty)}(x)$ are known for every $\lambda>0$ (cf. [7]), but the fact that $\sin (a \lambda) d \lambda$ is a signed measure introduces additional difficulties.

Definition 3. The Laguerre-Pólya class $L P$ consists of all entire functions of the form

$$
F(z)=C e^{-\gamma z^{2}+b z} z^{\kappa} \prod_{k=1}^{\infty}\left(1-\frac{z}{a_{k}}\right) e^{z / a_{k}}
$$


where $\gamma \geq 0, \kappa \in \mathbb{N}_{0}, b, C, a_{k}(k \in \mathbb{N})$ are real, and

$$
\sum_{k=1}^{\infty} \frac{1}{a_{k}^{2}}<\infty
$$

We denote by $\mathcal{T}_{F}$ the set of zeros of $F$. If the set of zeros is bounded above, we include $\infty \in \mathcal{T}_{F}$, if the set is bounded below, we include $-\infty \in \mathcal{T}_{F}$.

For $c \in \mathbb{R} \backslash \mathcal{T}_{F}$ we define

$$
g_{c}(t)=\frac{1}{2 \pi i} \int_{c-i \infty}^{c+i \infty} \frac{e^{s t}}{F(s)} d s,
$$

if this integral converges absolutely. (This is the case if $\gamma>0$ or if $F$ has at least two zeros.) Let $\tau_{1}$ and $\tau_{2}$ be the consecutive elements from $\mathcal{T}_{F}$ that satisfy $\tau_{1}<c<\tau_{2}$. A Fourier inversion shows that

$$
\frac{1}{F(z)}=\int_{-\infty}^{\infty} e^{-z t} g_{c}(t) d t
$$

in the strip $\tau_{1}<\Re z<\tau_{2}$. An application of the residue theorem shows that $g_{c}=g_{d}$ for $c, d \in\left(\tau_{1}, \tau_{2}\right)$.

As part of a series of papers on total positivity, I.J. Schoenberg 24 gave an intrinsic characterization of the functions $g$ that may occur as Laplace inverse transformations of LP functions. We require estimates that can be found in 12 .

Lemma 4. Let $F \in L P$ have at least $n+2$ zeros counted with multiplicity, and let $\tau_{1}, \tau_{2} \in \mathcal{T}_{F}$ with $0 \in\left(\tau_{1}, \tau_{2}\right)$. Then $g_{0}^{(k)}$ exists for $k \leq n$ and has at most $k$ sign changes on the real line.

Proof. This is [12, Chapter IV, Theorem 5.1].

Lemma 5. Let $F \in L P$ have at least two zeros. Let $\tau_{1}<\tau_{2}$ be two consecutive elements in $\mathcal{T}_{F}$, and let $c \in\left(\tau_{1}, \tau_{2}\right)$. Then there exist polynomials $P_{n}$ and $Q_{n}$ such that

$$
\begin{array}{r}
\left|g_{c}^{(n)}(t)\right| \leq\left|P_{n}(t)\right| e^{\tau_{1} t} \text { as } t \rightarrow \infty, \\
\left|g_{c}^{(n)}(t)\right| \leq\left|Q_{n}(t)\right| e^{\tau_{2} t} \text { as } t \rightarrow-\infty .
\end{array}
$$

Proof. Let $t>0$. The estimate follows from (2.3) by moving the integration path to $\Re s=d$ with $d<\tau_{1}$ using the residue theorem, see, e.g., [12, Chapter V, Theorem 2.1]. For $t<0$ the integration path is moved to $\Re s=d$ with $d>\tau_{2}$.

Lemma 6. Let $F$ be an even LP-function with a double zero at the origin and at least one positive zero $\tau$. Assume that $F$ is positive in $(0, \tau)$, and define $g=g_{\tau / 2}$ by (2.3).

(1) The derivatives $g^{\prime}$ and $g^{\prime \prime}$ exist and are nonnegative on the real line.

(2) The function $g^{\prime \prime}$ is even.

(3) If $-\tau<\Im \zeta<\tau$, then

$$
\int_{-\infty}^{\infty} g^{\prime \prime}(\lambda) \cos (\zeta \lambda) d \lambda=-\frac{\zeta^{2}}{F(i \zeta)}
$$

In particular, $F$ is real-valued on the imaginary axis. 
(4) If $-\tau<\Im \zeta<\tau$ and $w \in \mathbb{R}$, then

$$
\int_{-\infty}^{\infty} g^{\prime \prime}(\lambda+w) \sin (\zeta \lambda) d \lambda=\frac{\zeta^{2} \sin (\zeta w)}{F(i \zeta)} .
$$

Proof. Since $F$ has at least four zeros counted with multiplicity, (2.3) may be differentiated twice under the integral sign, which shows that $g^{\prime}$ and $g^{\prime \prime}$ exist. Two integration by parts show that

$$
\frac{z^{j}}{F(z)}=\int_{-\infty}^{\infty} e^{-z t} g^{(j)}(t) d t \quad(j \in\{0,1,2\})
$$

for all $z$ with $0<\Re z<\tau$. Since $z^{-j} F(z)$ is in $L P$ for $j \in\{0,1,2\}$, Lemma 4 with $k=0$ implies that $g^{(j)}$ has no sign changes on the real line. Evaluation of (2.8) at $z=\tau / 2$ shows that these derivatives are nonnegative on the real line. Since $z^{-2} F(z)$ has no zero at the origin, (2.8) extends to $-\tau<\Re z<\tau$ for $j=2$. Since $z^{-2} F(z)$ is an even function of $z,(2.3)$ with $c=0$ gives

$$
g^{\prime \prime}(t)=\frac{1}{2 \pi} \int_{-\infty}^{\infty} \frac{(i u)^{2} e^{i u t}}{F(i u)} d u
$$

which implies that $g^{\prime \prime}$ is even.

Let $\zeta$ such that $-\tau<\Im \zeta<\tau$. Since (2.8) extends to $-\tau<\Re z<\tau$, we have

$$
\int_{-\infty}^{\infty} g^{\prime \prime}(\lambda) \cos (\zeta \lambda) d \lambda=\frac{1}{2}\left(\frac{(i \zeta)^{2}}{F(i \zeta)}+\frac{(-i \zeta)^{2}}{F(-i \zeta)}\right)
$$

and (2.6) follows since $F$ is even. Since $\lambda \mapsto g^{\prime \prime}(\lambda) \sin \zeta \lambda$ is odd, the trigonometric identity $\sin \zeta(\lambda-w)=\cos \zeta w \sin \zeta \lambda-\cos \zeta \lambda \sin \zeta w$ implies

$$
\int_{-\infty}^{\infty} g^{\prime \prime}(\lambda) \sin \zeta(\lambda-w) d \lambda=-\sin \zeta w \int_{-\infty}^{\infty} g^{\prime \prime}(\lambda) \cos \zeta \lambda d \lambda .
$$

An application of (2.6) gives (2.7).

Define for positive $a$ the function $h_{a}: \mathbb{R} \rightarrow \mathbb{R}$ by

$$
h_{a}(w)=-\int_{-\infty}^{0} g(\lambda+w) \sin a \lambda d \lambda,
$$

and note that (2.5) with $\tau_{2}=\tau$ implies that $g(t)$ and $g^{\prime}(t)$ decay exponentially as $t \rightarrow-\infty$. Hence $h_{a}(w)$ is finite for every $w \in \mathbb{R}$, and an integration by parts gives for all real $w$

$$
h_{a}(w)=\frac{1}{a} \int_{-\infty}^{0} g^{\prime}(\lambda+w)(1-\cos a \lambda) d \lambda .
$$

We require bounds for the derivatives of $h_{a}$ and evaluations for special values.

Lemma 7. Let $a>0$. Let $F \in L P$ be even with a double zero at the origin, at least one positive zero $\tau$, and at least five zeros (counted with multiplicity). Assume that $F$ is positive in $(0, \tau)$, and define $g=g_{\tau / 2}$ by (2.3).

(1) The inequalities

$$
0 \leq h_{a}^{(n)}(w) \leq \frac{2}{a} g^{(n)}(w)
$$

hold for $n \in\{0,1\}$ and all real $w$, and for $n=2$ and $w \leq 0$. 
(2) We have the representation

$$
h_{a}^{\prime}(0)=\frac{g^{\prime}(0)}{a}+\frac{a}{2 F(i a)} .
$$

(3) For all real $w$ we have

$$
h_{a}^{\prime \prime}(w)-h_{a}^{\prime \prime}(-w)=-\sin (a w) \frac{a^{2}}{F(i a)} .
$$

Proof. Equation (2.10) implies for all $n$ and all real $w$

$$
h_{a}^{(n)}(w)=\int_{-\infty}^{0} g^{(n+1)}(\lambda+w) \frac{1-\cos (a \lambda)}{a} d \lambda .
$$

The functions $g, g^{\prime}$, and $g^{\prime \prime}$ are nonnegative on $\mathbb{R}$ by Lemma [6] and $g^{\prime \prime \prime}$ has exactly one change of sign on $\mathbb{R}$ by Lemma 4 applied to $g^{\prime \prime}$. Since $g^{\prime \prime}$ is even, the sign change is located at the origin. It follows that for all real $w$ and $n \in\{0,1\}$, as well as for $n=2$ and $w \leq 0$,

$$
0 \leq h_{a}^{(n)}(w) \leq \int_{-\infty}^{0} g^{(n+1)}(\lambda+w) \frac{2}{a} d \lambda=\frac{2}{a} g^{(n)}(w),
$$

which implies inequality (2.11).

To prove (2.12) we differentiate (2.9) and set $w=0$ to get

$$
h_{a}^{\prime}(0)=-\int_{-\infty}^{0} g^{\prime}(\lambda) \sin (a \lambda) d \lambda .
$$

We perform an integration by parts, apply that $g^{\prime \prime}$ is even, and use (2.6) to obtain

$$
\begin{aligned}
h_{a}^{\prime}(0) & =\frac{g^{\prime}(0)}{a}-\frac{1}{a} \int_{-\infty}^{0} g^{\prime \prime}(\lambda) \cos (a \lambda) d \lambda \\
& =\frac{g^{\prime}(0)}{a}-\frac{1}{2 a} \int_{-\infty}^{\infty} g^{\prime \prime}(\lambda) \cos (a \lambda) d \lambda \\
& =\frac{g^{\prime}(0)}{a}+\frac{a}{2 F(i a)}
\end{aligned}
$$

which finishes the proof of (2.12). Equations (2.9) and (2.7) give

$$
\begin{aligned}
h_{a}^{\prime \prime}(w) & =-\int_{-\infty}^{\infty} g^{\prime \prime}(\lambda+w) \sin (a \lambda) d \lambda+\int_{0}^{\infty} g^{\prime \prime}(\lambda+w) \sin (a \lambda) d \lambda \\
& =-\sin (a w) \frac{a^{2}}{F(i a)}+h_{a}^{\prime \prime}(-w)
\end{aligned}
$$

which is (2.13).

The next proposition investigates two interpolations of $t_{a}$ in $\Re z \geq 0$ and $\Re z \leq \tau$, respectively, and shows that they are representations of a single entire function $z \mapsto A(F, a, z)$ which interpolates $x \mapsto t_{a}(x-)$ at the zeros of $F$. See [7] for a similar construction for the cutoff of an exponential function. 
Proposition 8. Let $a>0$. Let $F \in L P$ be even with a double zero at the origin and at least one positive zero $\tau$. Assume that $F$ is positive in $(0, \tau)$, and define $g=g_{\tau / 2}$ by (2.3) and $h_{a}$ by (2.9). Define

$$
\begin{aligned}
& A_{1}(F, a, z)=\frac{F(z)}{a} \int_{-\infty}^{0} h_{a}(w) e^{-z w} d w \text { for } \Re z<\tau \\
& A_{2}(F, a, z)=\frac{1}{z^{2}+a^{2}}-\frac{F(z)}{a} \int_{0}^{\infty} h_{a}(w) e^{-z w} d w \text { for } \Re z>0 .
\end{aligned}
$$

Then $z \mapsto A_{1}(F, a, z)$ is analytic in $\Re z<\tau, z \mapsto A_{2}(F, a, z)$ is analytic in $\Re z>0$, and these functions are restrictions of an entire function $z \mapsto A(F, a, z)$. Moreover, there exists a constant $c>0$ so that

$$
|A(F, a, z)| \leq c(1+|F(z)|)
$$

for all $z \in \mathbb{C}$.

Proof. Inequality (2.5) with $\tau_{2}=\tau$ implies that $g^{\prime}(t)$ decays exponentially as $t \rightarrow$ $-\infty$. Hence it follows from (2.10) that

$$
0 \leq h_{a}(w) \leq \frac{2}{a} g(w)
$$

for all real $w$, and (2.5) with $\tau_{2}=\tau$ applied to $g$ for $t \rightarrow-\infty$ implies that the integral defining $A_{1}(F, a, z)$ converges absolutely in $\Re z<\tau$. Inequality (2.5) implies with $\tau_{1}=0$ that $g$ has polynomial growth on the positive real axis, hence the integral in the definition of $A_{2}(F, a, z)$ converges absolutely for $\Re z>0$. It follows that $A_{1}$ and $A_{2}$ are analytic functions in $\Re z>\tau$ and $0<\Re z$, respectively.

To prove that $A_{1}$ and $A_{2}$ are analytic continuations of each other it suffices to prove that they are equal in the strip $0<\Re z<\tau$. Starting point is the identity

$$
\frac{a}{z^{2}+a^{2}}=\int_{0}^{\infty} e^{-z \lambda} \sin a \lambda d \lambda,
$$

valid for $\Re z>0$. Combining this with (2.4) gives for $0<\Re z<\tau$

$$
\begin{aligned}
\frac{1}{z^{2}+a^{2}} & =\frac{F(z)}{a} \int_{-\infty}^{\infty} \int_{0}^{\infty} e^{-z(w+\lambda)} g(w) \sin (a \lambda) d \lambda d w \\
& =\frac{F(z)}{a} \int_{-\infty}^{\infty} \int_{0}^{\infty} e^{-z w} g(w-\lambda) \sin (a \lambda) d \lambda d w \\
& =\frac{F(z)}{a} \int_{-\infty}^{\infty} h_{a}(w) e^{-z w} d w .
\end{aligned}
$$

Inserting this in (2.14) shows that $A_{1}(F, a, z)=A_{2}(F, a, z)$ for $0<\Re z<\tau$. To prove (2.15) we note that inequality (2.16) implies in $\Re z \leq \tau / 2$

$$
|A(F, a, z)| \leq \frac{|F(z)|}{a} \int_{-\infty}^{0}\left|h_{a}(w) e^{-z w}\right| d w \leq \frac{2|F(z)|}{a^{2}} \int_{-\infty}^{0} g(w) e^{-\tau w / 2} d w,
$$

and an analogous calculation holds in $\Re z \geq \tau / 2$.

Starting with the function $A(F, a, z)$, we construct interpolations $M^{ \pm}$of $t_{a}$ that interpolate $t_{a}$ at the zeros of $F$ (with correct multiplicity) so that $M^{ \pm}-t_{a}$ has no sign changes between two consecutive zeros of $F$. This is accomplished by selecting the value at the origin appropriately. We assume that $a>0$, and that $F \in L P$ and 
$\tau>0$ satisfy the assumptions of Proposition 8. We define $z \mapsto M^{+}(F, a, z)$ and $z \mapsto M^{-}(F, a, z)$ by

$$
\begin{aligned}
& M^{-}(F, a, z)=A(F, a, z)+\frac{h_{a}(0)}{a} \frac{F(z)}{z} \\
& M^{+}(F, a, z)=A(F, a, z)+\frac{h_{a}(0)}{a} \frac{F(z)}{z}+\frac{2 g^{\prime}(0)}{a^{2}} \frac{F(z)}{z^{2}}
\end{aligned}
$$

where $A(F, a, z)$ is defined in (2.14). Evidently $M^{+}$and $M^{-}$are entire functions. Recall that $\mathcal{T}_{F} \cap \mathbb{R}$ is the zero set of $F$. It is evident from the definitions that $M^{ \pm}(F, a, \xi)=t_{a}(\xi)$ for all real $\xi \in \mathcal{T}_{F} \backslash\{0\}$. Since $F$ has a double zero at the origin, we see that $M^{-}(F, a, 0)=0$.

Since $g^{\prime \prime}$ is nonnegative and integrable on $\mathbb{R},(2.8)$ implies

$$
\frac{2}{F^{\prime \prime}(0)}=\int_{-\infty}^{\infty} g^{\prime \prime}(w) d w
$$

As $g^{\prime \prime}$ is even and $g^{\prime}(w)$ decays exponentially as $w \rightarrow-\infty$, we also have that $\int_{-\infty}^{\infty} g^{\prime \prime}(w) d w=2 g^{\prime}(0)$. Hence, $z^{-2} F(z) \rightarrow 1 /\left(2 g^{\prime}(0)\right)$ as $z \rightarrow 0$ and $M^{+}(F, a, 0)=$ $1 / a^{2}$. This means that

$$
M^{ \pm}(F, a, \xi)=t_{a}(\xi \pm)
$$

for all real $\xi \in \mathcal{T}_{F}$, where $t_{a}(\xi \pm)$ denotes the one sided limits at $\xi$.

Theorem 9. Let $a>0$. Let $F \in L P$ be even with a double zero at the origin and at least one positive zero $\tau$. Assume that $F$ is positive in $(0, \tau)$, and define $g=g_{\tau / 2}$ by (2.3) and $h_{a}$ by (2.9). If

$$
F(i a)<0,
$$

then

$$
F(x)\left\{M^{+}(F, a, x)-t_{a}(x)\right\} \geq 0
$$

for all real $x$.

Proof. Consider first $x<0$. An expansion of the second term in (2.20) in a Laplace transform together with (2.14) gives

$$
M^{+}(F, a, x)-t_{a}(x)=\frac{F(x)}{a} \int_{-\infty}^{0}\left(h_{a}(w)-h_{a}(0)\right) e^{-x w} d w+\frac{F(x)}{x^{2}} \frac{2 g^{\prime}(0)}{a^{2}} .
$$

Two integration by parts and (2.12) lead to

$$
\begin{aligned}
\int_{-\infty}^{0}\left(h_{a}(w)-h_{a}(0)\right) e^{-x w} d w & =\frac{1}{x^{2}} \int_{-\infty}^{0} h_{a}^{\prime \prime}(w) e^{-w x} d x-\frac{h_{a}^{\prime}(0)}{x^{2}} \\
& =\frac{1}{x^{2}} \int_{-\infty}^{0} h_{a}^{\prime \prime}(w) e^{-x w} d w-\frac{g^{\prime}(0)}{a x^{2}}-\frac{a}{2 x^{2} F(i a)},
\end{aligned}
$$

and inserting this in (2.23) gives

$$
M^{+}(F, a, x)-t_{a}(x)=\frac{F(x)}{x^{2}}\left(\frac{g^{\prime}(0)}{a^{2}}-\frac{1}{2 F(i a)}+\frac{1}{a} \int_{-\infty}^{0} h_{a}^{\prime \prime}(w) e^{-x w} d w\right) .
$$

By assumption $-F(i a)>0$, and (2.11) implies $h_{a}^{\prime \prime}(w) \geq 0$. Since by assumption $z^{-1} F(z)$ is a LP-function that is positive in $(0, \tau)$, it follows from Lemma 6 that $g^{\prime}(0)>0$. Hence (2.22) is shown for $x<0$. 
Let $x>0$. From (2.14), (2.9), and (2.12) we get

$$
\begin{aligned}
M^{+}(F, a, x)-t_{a}(x) & =-\frac{F(x)}{a} \int_{0}^{\infty} h_{a}(w) e^{-x w} d w+\frac{F(x)}{a} \frac{h_{a}(0)}{x}+\frac{F(x)}{x^{2}} \frac{2 g^{\prime}(0)}{a^{2}} \\
& =-\frac{F(x)}{a} \int_{0}^{\infty}\left(h_{a}(w)-h_{a}(0)\right) e^{-x w} d w+\frac{F(x)}{x^{2}} \frac{2 g^{\prime}(0)}{a^{2}},
\end{aligned}
$$

and, analogously to (2.24), we obtain the representation

$$
M^{+}(F, a, x)-t_{a}(x)=\frac{F(x)}{x^{2}}\left(\frac{g^{\prime}(0)}{a^{2}}-\frac{1}{2 F(i a)}-\frac{1}{a} \int_{0}^{\infty} h_{a}^{\prime \prime}(w) e^{-x w} d w\right)
$$

for $x>0$. In order to investigate the sign of the right hand side, we multiply (2.13) by $e^{-x w}$ and integrate $w$ over $[0, \infty)$ to get with (2.17)

$$
\begin{aligned}
\int_{0}^{\infty}\left(h_{a}^{\prime \prime}(w)-h_{a}^{\prime \prime}(-w)\right) e^{-x w} d w & =-\frac{a^{2}}{F(i a)} \int_{0}^{\infty} e^{-x w} \sin (a w) d w \\
& =-\frac{a^{2}}{F(i a)} \frac{a}{x^{2}+a^{2}} .
\end{aligned}
$$

Hence

$$
-\frac{1}{a} \int_{0}^{\infty} h_{a}^{\prime \prime}(w) e^{-x w} d w=\frac{a^{2}}{F(i a)} \frac{1}{x^{2}+a^{2}}-\frac{1}{a} \int_{0}^{\infty} h_{a}^{\prime \prime}(-w) e^{-x w} d w .
$$

Since $h_{a}^{\prime \prime}(-w) \geq 0$ for $w \geq 0$, we have from (2.12)

$$
\int_{0}^{\infty} h_{a}^{\prime \prime}(-w) e^{-x w} d w \leq \int_{0}^{\infty} h_{a}^{\prime \prime}(-w) d w=\frac{g^{\prime}(0)}{a}+\frac{a}{2 F(i a)} .
$$

We multiply (2.27) by $-a^{-1}$ and insert the resulting inequality into (2.26) to get

$$
-\frac{1}{a} \int_{0}^{\infty} h_{a}^{\prime \prime}(w) e^{-x w} d w \geq \frac{a^{2}}{F(i a)} \frac{1}{x^{2}+a^{2}}-\frac{g^{\prime}(0)}{a^{2}}-\frac{1}{2 F(i a)} .
$$

Inserting this into (2.25) gives

$$
\frac{M^{+}(F, a, x)-t_{a}(x)}{F(x)} \geq \frac{1}{F(i a)} \frac{1}{x^{2}}\left(\frac{1}{(x / a)^{2}+1}-1\right),
$$

which is nonnegative since $F(i a)<0$. This proves (2.22) for $x>0$.

Theorem 10. Let $a>0$. Let $F \in L P$ be even with a double zero at the origin and at least one positive zero $\tau$. Assume that $F$ is positive in $(0, \tau)$, and define $g=g_{\tau / 2}$ by (2.3) and $h_{a}$ by (2.9). Then

$$
F(x)\left\{M^{-}(F, a, x)-t_{a}(x)\right\} \leq 0
$$

holds for all real $x$.

Proof. We note that the integral representations for $M^{+}$are valid even if $F(i a)$ is not negative. From the definition of $M^{-}$and (2.23) we obtain for $x<0$ the representation

$$
M^{-}(F, a, x)-t_{a}(x)=\frac{F(x)}{a} \int_{-\infty}^{0}\left(h_{a}(w)-h_{a}(0)\right) e^{-x w} d w,
$$


and since $h_{a}^{\prime}(w) \geq 0$ for real $w$, it follows that $h_{a}(w)-h_{a}(0) \leq 0$ for $w \leq 0$ which shows (2.28) for $x<0$. Analogously, for $x>0$

$$
M^{-}(F, a, x)-t_{a}(x)=-\frac{F(x)}{a} \int_{-\infty}^{0}\left(h_{a}(w)-h_{a}(0)\right) e^{-x w} d w,
$$

which gives (2.28) in this range.

Proposition 11. The functions $M^{+}$and $M^{-}$from Theorems 9 and 10 satisfy

$$
\left|M^{ \pm}(F, a, x)-t_{a}(x)\right|=\mathcal{O}\left(\frac{F(x)}{1+x^{2}}\right)
$$

for all real $x$.

Proof. Inequalities (2.23) and (2.25) yield (2.31) for $M^{+}$, while (2.29) and (2.30) imply (2.31) for $M^{-}$.

\section{Extremal functions for $t_{a}$ in DE Branges Spaces}

In this section we prove Theorem 1 . Recall that $E$ is an HB function that satisfies (I) $E$ is of bounded type in $\mathbb{C}^{+}$with mean type $\tau$, (II) $E$ has no real zeros, (III) $E^{*}(z)=E(-z)$ for all $z$, (IV) $B \notin \mathcal{H}(E)$. These conditions imply that $E$ has certain properties that we collect in the following lemma. We define the positive Borel measure $\mu_{E}$ by

$$
\mu_{E}(A)=\int_{A} \frac{d x}{|E(x)|^{2}} .
$$

Lemma 12. If $E$ is an $H B$ function satisfying (I) - (IV), then

(1) E has exponential type $\tau$,

(2) Every nonnegative $F \in \mathcal{A}_{1}\left(2 \tau, \mu_{E}\right)$ can be factored as $F=U U^{*}$ with $U \in$ $\mathcal{H}(E)$

(3) $A=(1 / 2)\left(E+E^{*}\right)$ is even and $B=(i / 2)\left(E-E^{*}\right)$ is odd,

(4) For every $U \in \mathcal{H}(E)$ the identity

$$
\int_{-\infty}^{\infty}\left|\frac{U(x)}{E(x)}\right|^{2} d x=\sum_{\xi \in \mathcal{T}_{B}} \frac{|U(\xi)|^{2}}{K(\xi, \xi)}
$$

is valid. (Recall that $\mathcal{T}_{B}$ is the set of zeros of $B$.)

Proof. Since $E$ is of bounded type with mean type $\tau$ in $\mathbb{C}^{+}$, it follows from (1.2) that $E^{*}$ is of bounded type with mean type $\leq \tau$ in $\mathbb{C}^{+}$. It follows from Krein's theorem 22, Theorems 6.17 and 6.18] that $E$ has exponential type $\tau$. The second property follows from [17, Appendix V] together with the observation that $F \in \mathcal{A}_{1}\left(2 \tau, \mu_{E}\right)$ implies

$$
\int_{-\infty}^{\infty} \frac{\log ^{+}|F(x)|}{1+x^{2}} d x<\infty
$$

This follows from Jensen's inequality, see, e.g., the proof of [13, Lemma 12]. The third property is evident, and (3.2) is [4, Theorem 22].

Proof of Theorem 1, Assume that $E$ satisfies (I) - (IV). Inequality (1.2) implies that $A$ and $B$ have only real zeros, and since $E$ has no real zeros, it follows that $A$ and $B$ have no common zeros. By [4, Problem 48] there exists a continuous increasing function $\varphi$ such that $E(x) \exp (i \varphi(x))$ is real valued for all real $x$. The 
zeros of $A$ are the values $x$ where $\varphi(x)=\frac{\pi}{2}+k \pi$ for some $k \in \mathbb{Z}$, and the zeros of $B$ are the values $x$ where $\varphi(x)=k \pi$ for some $k \in \mathbb{Z}$. It follows that the zeros interlace. It follows from [4, Problem 47] applied to $f=A / B$ that the zeros of $B$ are simple (see also the proof of Theorem 22 in [4]). Similarly, the zeros of $A$ are simple.

Since $E$ has exponential type $\tau$, it follows that $A$ and $B$ are also entire functions of exponential type $\tau$. Hence they, and their squares, are Laguerre-Pólya entire functions. Evidently, $B^{2}$ is an even LP function that has a double zero at the origin, and the results of Section 2 are applicable.

We define the entire functions $T_{a}^{+}$and $T_{a}^{-}$by

$$
\begin{aligned}
& T_{a}^{+}(z)=M^{+}\left(B^{2}, a, z\right) \\
& T_{a}^{-}(z)=M^{-}\left(B^{2}, a, z\right)
\end{aligned}
$$

with $M^{-}$and $M^{+}$as in (2.19) and (2.20).

We note that $E^{*}(z)=E(-z)$ implies in particular that $\overline{B(i x)}=-B(i x)$ for real $x$, i.e., $\Re B(i x)=0$ for real $x$. It follows then that

$$
(B(i x))^{2}<0
$$

for every real $x$, hence $F=B^{2}$ satisfies the assumptions of Theorem 9 , Since $B^{2} \geq 0$ on $\mathbb{R}$, inequality (2.22) implies

$$
T_{a}^{+}(x) \geq t_{a}(x)
$$

for all real $x$, and (2.21) implies that

$$
T_{a}^{+}(\xi)=t_{a}(\xi)
$$

for all $\xi$ with $B(\xi)=0$. Since $B^{2} / E^{2}$ is bounded on $\mathbb{R}$, it follows from (2.31) that

$$
\int_{-\infty}^{\infty} \frac{T_{a}^{+}(x)-t_{a}(x)}{|E(x)|^{2}} d x<\infty .
$$

A similar argument gives the same statement for $t_{a}-T_{a}^{-}$. Since $T_{a}^{-} \leq t_{a} \leq T_{a}^{+}$ we obtain that $\left|T_{a}^{+}-T_{a}^{-}\right|$is integrable with respect to $\mu_{E}=|E(x)|^{-2} d x$. It follows from Lemma 12 that there exists $U \in \mathcal{H}(E)$ such that

$$
T_{a}^{+}-T_{a}^{-}=U U^{*} .
$$

We prove next the optimality of $T_{a}^{+}$. Let $F$ be a function of type $2 \tau$ with $F \geq t_{a}$ on $\mathbb{R}$. We may assume that

$$
\int_{-\infty}^{\infty} \frac{F(x)-t_{a}(x)}{|E(x)|^{2}} d x<\infty
$$

(since otherwise there is nothing to show). The inequality $T_{a}^{-} \leq t_{a} \leq F$ gives

$$
\left|F(x)-T_{a}^{-}(x)\right| \leq\left(F(x)-t_{a}(x)\right)+\left(t_{a}(x)-T_{a}^{-}(x)\right)
$$

and hence $F-T_{a}^{-}$is an entire function of exponential type $2 \tau$ that is integrable with respect to $\mu_{E}$. Evidently, $F-T_{a}^{-} \geq F-t_{a} \geq 0$. Applying Lemma 12 again implies that there exists $V \in \mathcal{H}(E)$ such that

$$
F-T_{a}^{-}=V V^{*},
$$

It follows from (3.3) and (3.4) that

$$
F-T_{a}^{+}=V V^{*}-U U^{*} .
$$


An application of Lemma 12 (4) to $U$ and $V$ together with $T_{a}^{+}(\xi)=t_{a}(\xi)$ for all $\xi \in \mathbb{R}$ with $B(\xi)=0$ implies

$$
\int_{-\infty}^{\infty} \frac{F(x)-T_{a}^{+}(x)}{|E(x)|^{2}} d x=\sum_{B(\xi)=0} \frac{F(\xi)-t_{a}(\xi)}{K(\xi, \xi)} \geq 0,
$$

hence $T_{a}^{+}$is extremal.

An analogous calculation (which we omit) shows that $T_{a}^{-}$is an extremal minorant. It remains to prove that

$$
\int_{-\infty}^{\infty}\left(T_{a}^{+}(x)-T_{a}^{-}(x)\right) \frac{d x}{|E(x)|^{2}}=\frac{1}{a^{2} K(0,0)} .
$$

It follows from (3.3) and Lemma 12 (4) that

$$
\int_{-\infty}^{\infty}\left(T_{a}^{+}(x)-T_{a}^{-}(x)\right) \frac{d x}{|E(x)|^{2}}=\sum_{B(\xi)=0} \frac{T_{a}^{+}(\xi)-T_{a}^{-}(\xi)}{K(\xi, \xi)} .
$$

The only non-zero summand is the term for $\xi=0$. Since $T_{a}^{+}(0)-T_{a}^{-}(0)=$ $t_{a}(0)-t_{a}(0-)=1 / a^{2}$, the proof is complete.

\section{De Branges Space and optimal functions for the VANishing CONDITION}

Define the Borel measure $\mu_{a}$ by

$$
\mu_{a}(B)=\int_{B}\left(x^{2}+a^{2}\right) d x
$$

Let $a>0$ and recall that $E_{a}$ is given by

$$
E_{a}(z)=\sqrt{\frac{2}{\sinh (2 \pi a)}} \frac{\sin \pi(z+i a)}{(z+i a)} .
$$

We prove that $E_{a}$ is a Hermite-Biehler function whose associated de Branges space is isometrically equal to $\mathcal{A}_{2}\left(\pi, \mu_{a}\right)$.

Theorem 13. Let $a>0$. The function $E_{a}$ satisfies (1.2) and properties (I) - (IV). Moreover, the space $\mathcal{A}_{2}\left(\pi, \mu_{a}\right)$ is isometrically equal to $\mathcal{H}\left(E_{a}\right)$.

Proof. Since $z \mapsto \sin \pi z$ is LP and hence of Pólya class, we have that $z \mapsto \sin (\pi(z+$ $i a)$ ) is also of Pólya class. By [4, Section 7, Lemma 1] it follows that $E_{a}$ is of Pólya class. This implies

$$
\left|E_{a}(z)\right| \geq\left|E_{a}^{*}(z)\right|
$$

for all $z$ with $\Im z>0$. Since $E_{a}$ has no zeros in the upper half plane, the function $E_{a}^{*} / E_{a}$ is analytic in the upper half plane and has modulus bounded by 1 . Since this quotient is not constant, the modulus is never equal to 1 by the maximum principle, hence $E_{a}$ satisfies (1.2).

It can be checked directly that $E_{a}$ has bounded type $\pi$ (or apply the reverse direction of Krein's theorem). Evidently $E_{a}$ has no real zeros and $E_{a}^{*}(z)=E_{a}(-z)$ 
for all $z$. A direct calculation gives

$$
\begin{aligned}
& A_{a}(z)=\sqrt{\frac{2}{\sinh (2 \pi a)}} \frac{z \cosh (\pi a) \sin (\pi z)+a \sinh (\pi a) \cos (\pi z)}{z^{2}+a^{2}}, \\
& B_{a}(z)=\sqrt{\frac{2}{\sinh (2 \pi a)}} \frac{a \cosh (\pi a) \sin (\pi z)-z \sinh (\pi a) \cos (\pi z)}{z^{2}+a^{2}},
\end{aligned}
$$

and in particular $B_{a} \notin \mathcal{H}\left(E_{a}\right)$. Hence $E_{a}$ satisfies (I) - (IV).

Taking limits in (1.3) leads to the representation

$$
K_{a}(x, x)=\frac{\pi\left(a^{2}+x^{2}\right)-a \operatorname{coth}(2 \pi a)+a \cos (2 \pi x) \operatorname{csch}(2 \pi a)}{\pi\left(a^{2}+x^{2}\right)^{2}}
$$

for all real $x$. Recall that $\mu_{E_{a}}(A)=\int_{A}\left|E_{a}(x)\right|^{-2} d x$. It is straightforward to check that $L^{2}\left(\mathbb{R}, \mu_{a}\right)$ and $L^{2}\left(\mathbb{R}, \mu_{E_{a}}\right)$ are equal as sets with equivalent norms. It follows that $\mathcal{A}_{2}\left(\pi, \mu_{a}\right)$ and $H\left(E_{a}\right)$ are equal as sets and have equivalent norms. The main statement to prove is the fact that the two norms are equal on the smaller spaces.

We note first that

$$
\frac{(z+i a)(z-i a)}{\sin (\pi(z+i a)) \sin (\pi(z-i a))}=\frac{2\left(z^{2}+a^{2}\right)}{\cosh (2 \pi a)-\cos (2 \pi z)}
$$

holds, in particular, the right hand side is 1-periodic after division by $z^{2}+a^{2}$. Furthermore,

$$
\int_{0}^{1} \frac{1}{\cosh (2 \pi a)-\cos (2 \pi x)} d x=\frac{1}{\sinh (2 \pi a)} .
$$

This means that $p_{a}$ defined by $p_{a}(x)=\sinh (2 \pi a)(\cosh (2 \pi a)-\cos (2 \pi x))^{-1}-1$ is 1-periodic and has mean value zero. Since $a>0$, this function is infinitely differentiable on the real line. It follows that the Fourier series of $p_{a}$ converges absolutely and uniformly, and that it represents the function, i.e., there exists a sequence $a_{n}$ so that

$$
p_{a}(x)=\sum_{n \neq 0} a_{n} e^{2 \pi i n x}
$$

for all real $x$.

Let $H \in L^{1}(\mathbb{R})$ be an entire function of exponential type $2 \pi$. Since $H \in L^{2}(\mathbb{R})$ by [6, Theorem 6.7.1], the Paley-Wiener theorem [25, Theorem 4.1] implies that the Fourier transform $\widehat{H}$ defined by

$$
\widehat{H}(t)=\int_{-\infty}^{\infty} e^{-2 \pi i x t} H(x) d x
$$

satisfies $\widehat{H}(t)=0$ for $|t|>1$. Since $H \in L^{1}(\mathbb{R})$ it follows that $\widehat{H}$ is continuous, hence $\widehat{H}(t)=0$ for $|t| \geq 1$. This implies

$$
\int_{-\infty}^{\infty} H(x) \sum_{\substack{|n| \leq N \\ n \neq 0}} a_{n} e^{2 \pi i n x} d x=\sum_{\substack{|n| \leq N \\ n \neq 0}} a_{n} \widehat{H}(-n)=0 .
$$


Since the partial sums of the series in 4.3) converge uniformly, we obtain with an application of Lebesgue dominated convergence that

$$
\int_{-\infty}^{\infty} H(x)\left(\frac{\sinh (2 \pi a)}{\cosh (2 \pi a)-\cos (2 \pi x)}-1\right) d x=0
$$

Let $F, G \in \mathcal{A}_{2}\left(\pi, \mu_{a}\right)$ and define $H$ by $H(z)=F(z) G^{*}(z)\left(z^{2}+a^{2}\right)$. It follows from (4.2) that

$$
\begin{aligned}
\langle F, G\rangle_{\mathcal{H}\left(E_{a}\right)}-\langle F, G\rangle_{L^{2}\left(\mathbb{R}, \mu_{a}\right)} & =\int_{-\infty}^{\infty} F(x) G^{*}(x)\left\{\left|E_{a}(x)\right|^{-2}-\left(x^{2}+a^{2}\right)\right\} d x \\
& =\int_{-\infty}^{\infty} H(x)\left(\frac{\sinh (2 \pi a)}{\cosh (2 \pi a)-\cos (2 \pi x)}-1\right) d x
\end{aligned}
$$

and since $H$ is a Lebesgue integrable entire function of exponential type $2 \pi$, it follows from (4.4) that

$$
\langle F, G\rangle_{\mathcal{H}\left(E_{a}\right)}=\langle F, G\rangle_{L^{2}\left(\mathbb{R}, \mu_{a}\right)}
$$

as claimed.

Sketch of an alternate proof. Define for $a>0$ the meromorphic function $W_{a}$ by

$$
W_{a}(z)=-e^{-2 \pi a} \frac{a+i z}{a-i z}
$$

and note that $W_{a}$ is analytic and has modulus $\leq 1$ in the upper half plane. The identity

$$
\frac{E_{a}(z)+E_{a}^{*}(z) W_{a}(z)}{E_{a}(z)-E_{a}^{*}(z) W_{a}(z)}=\operatorname{coth}(2 \pi a)-e^{2 \pi i z} \operatorname{csch}(2 \pi a)
$$

is valid for $z \in \mathbb{C}$, and for real $x$ and $y>0$

$$
\frac{y}{\pi} \int_{-\infty}^{\infty} \frac{\left(t^{2}+a^{2}\right)\left|E_{a}(t)\right|^{2} d t}{(x-t)^{2}+y^{2}}=\operatorname{coth}(2 \pi a)-e^{-2 \pi y} \cos (2 \pi x) \operatorname{csch}(2 \pi a)
$$

holds. Theorem V.A of [5] with $d \mu(t)=\left(t^{2}+a^{2}\right)\left|E_{a}(t)\right|^{2} d t$ implies

$$
\int_{-\infty}^{\infty}|f(x)|^{2}\left(x^{2}+a^{2}\right) d x=\int_{-\infty}^{\infty}\left|\frac{f(x)}{E_{a}(x)}\right|^{2} d x
$$

for every $f \in \mathcal{H}\left(E_{a}\right)$.

Proof of Theorem 2. We define $S_{a}^{+}$and $S_{a}^{-}$by

$$
\begin{aligned}
& S_{a}^{+}(z)=M^{+}\left(B_{a}^{2}, a, z\right)\left(z^{2}+a^{2}\right), \\
& S_{a}^{-}(z)=M^{-}\left(B_{a}^{2}, a, z\right)\left(z^{2}+a^{2}\right) .
\end{aligned}
$$

Since $B_{a}$ is odd, we have $B_{a}(0)=0$. Since $E_{a}$ is Hermite-Biehler and is of bounded type in the upper half plane, it follows that $B_{a}$ is of bounded type in the upper half plane. Since $B_{a}$ has only real zeros, 4, Problem 34] implies that $B_{a}$ is of Pólya class, and [4, Theorem 7] implies that $B_{a} \in L P$. Hence $F=B_{a}^{2}$ satisfies the assumptions of Proposition 8 . Furthermore, it can be checked directly that $B_{a}^{2}(i a)<0$. For the remainder of the proof we set $M^{ \pm}(z)=M^{ \pm}\left(B_{a}^{2}, a, z\right)$. Since $B_{a}^{2} \in \mathcal{A}(2 \pi)$ we obtain that $M^{+}, M^{-} \in \mathcal{A}(2 \pi)$. Theorems 9 and 10 imply

$$
M^{-}(x) \leq t_{a}(x) \leq M^{+}(x)
$$


for all real $x$. The proof of Theorem 1 shows that $M^{+}=T_{a}^{+}$and $M^{-}=T_{a}^{-}$with respect to the measure $d \mu_{E_{a}}$. It follows from (1.5) that

$$
\int_{-\infty}^{\infty}\left(M^{+}(x)-M^{-}(x)\right) \frac{d x}{\left|E_{a}(x)\right|^{2}}=\frac{1}{a^{2} K_{a}(0,0)}=\frac{\pi a}{\pi a-\tanh (\pi a)} .
$$

By definition of $S_{a}^{ \pm}$we have

$$
\int_{-\infty}^{\infty}\left(S_{a}^{+}(z)-S_{a}^{-}(x)\right) d x=\int_{-\infty}^{\infty}\left(M^{+}(x)-M^{-}(x)\right)\left(x^{2}+a^{2}\right) d x .
$$

Since $M^{+}-M^{-} \in \mathcal{A}_{1}\left(2 \pi, \mu_{a}\right)$, it follows that $M^{+}-M^{-} \in \mathcal{A}_{1}\left(2 \pi, \mu_{E_{a}}\right)$. Lemma 12 (2) implies that $M^{+}-M^{-}=U U^{*}$ with $U \in \mathcal{H}\left(E_{a}\right)$. Theorem 13, (4.5), and (4.6) imply

$$
\int_{-\infty}^{\infty}\left(S_{a}^{+}(x)-S_{a}^{-}(x)\right) d x=\frac{\pi a}{\pi a-\tanh (\pi a)},
$$

which gives the case of equality in (1.7).

Let now $S, T \in \mathcal{A}(2 \pi)$ such that $S(i a)=T(i a)=0$ and $S(x) \leq t_{a}(x) \leq T(x)$ on the real line. We may assume that $S-M^{-}$and $T-M^{+}$are integrable with respect to $\left(x^{2}+a^{2}\right) d x$. Since $S$ and $T$ are real entire, it follows that $S(-i a)=T(-i a)=0$, hence

and

$$
S(z)=\left(z^{2}+a^{2}\right) \sigma(z)
$$

$$
T(z)=\left(z^{2}+a^{2}\right) \tau(z)
$$

where $\sigma, \tau$ are entire and have exponential type $2 \pi$. Furthermore, $\sigma-t_{a}$ and $\tau-t_{a}$ are integrable and

$$
\sigma(x) \leq t_{a}(x) \leq \tau(x)
$$

for all real $x$. It follows from Theorem 1 that

$$
\int_{-\infty}^{\infty}(\sigma(x)-\tau(x))\left(x^{2}+a^{2}\right) d x \geq \frac{1}{a^{2} K_{a}(0,0)}
$$

which is (1.7).

\section{REFERENCES}

[1] A.G. Babenko, Yu.V. Kryakin, V.A. Yudin, One-sided approximation in $L$ of the characteristic function of an interval by trigonometric polynomials, Proc. Steklov. Inst. Math. 280 (2013), S39 - S52.

[2] A. Beurling, On functions with a spectral gap, Seminar on Harmonic Analysis, Univ. of Uppsala. March 24, 1942. Published in Collected Works of Arne Beurling: Vol. II, Harmonic Analysis, Birkhäuser, Boston, 1989, 359-364.

[3] J. Bustamante, J.M. Quesada, R. Martinez-Cruz, Best one-sided $L_{1}$ approximation to the Heaviside and sign functions, J. Approx. Theory 164 (2012), $791-802$.

[4] L. de Branges, Hilbert spaces of entire functions, Prentice-Hall.

[5] L. de Branges, Some Hilbert spaces of entire functions, Trans. Amer. Math. Soc. 96, (1960), 259-295.

[6] R.P.B. Boas, Entire functions, Academic Press, New York 1954.

[7] E. Carneiro and F. Littmann, Extremal functions in de Branges and Euclidean spaces. Preprint.

[8] E. Carneiro, F. Littmann, J.D. Vaaler, Gaussian subordination and the Beurling-Selberg extremal problem, Trans. Amer. Math. Soc. 365 (2013), 3493-3534.

[9] D.L. Donoho, B.F. Logan, Signal recovery and the large sieve, SIAM J. Appl. Math. 52 no. 2 (1992), $577-591$.

[10] P.X. Gallagher, Pair correlation of zeros of the zeta function, J. Reine Angew. Math. 362 (1985), $72-86$ 
[11] M. Ganzburg, L-approximation to non-periodic functions, J. Concr. Appl. Math. 8 (2010), no. $2,208-215$.

[12] I.I. Hirschman and D.V. Widdder, The convolution transform, Dover.

[13] J. Holt and J. D. Vaaler, The Beurling-Selberg extremal functions for a ball in the Euclidean space, Duke Mathematical Journal 83 (1996), 203-247.

[14] M. Kelly, A variation on Selberg's approximation problem. Mathematika (http://dx.doi.org/10.1112/S0025579314000199)

[15] M.G. Krein, A contribution to the theory of entire functions of exponential type, Bull. Acad. Sci. URSS. Sèr. Math. 11 (1947), 309 - 326

[16] N. Korneichuk, Exact constants in approximation theory, Cambridge University Press, Cambridge, 1991.

[17] B.Ja. Levin, Distribution of zeros of entire functions, Translations of Mathematical Monographs 5, American Mathematical Society 1980.

[18] F. Littmann, Quadrature and extremal bandlimited functions, SIAM J. Math. Anal. 45 (2013), $732-747$.

[19] D.S. Lubinsky, Best approximation and interpolation of $\left(1+(a x)^{2}\right)^{-1}$ and its transforms, J. Approx. Theory 125 (2003), 106 - 115.

[20] D.S. Lubinsky, A survey of weighted polynomial approximation with exponential weights, Surveys in Approximation Theory 3 (2007), 1 - 106.

[21] A. Pinkus, On $L^{1}$-approximation, Cambridge University Press, 1989.

[22] M. Rosenblum and J. Rovnyak, Topics in Hardy classes and univalent functions, Birkh'auser Verlag, Basel 1994.

[23] A. Selberg, Lectures on sieves, Collected works Vol. 2., $65-249$.

[24] I.J. Schoenberg, On Pólya frequency functions. I. The totally positive functions and their Laplace transforms, J. Analyse Math. 1, (1951), 331 - 374.

[25] E.M. Stein and G. Weiss, Introduction to Fourier analysis on Euclidean spaces, Princeton University Press, New Jersey, 1971.

[26] J.D. Vaaler, Some extremal functions in Fourier analysis, Bull. Amer. Math. Soc. 12, no. 2 (1985), $183-215$.

Department of mathematics, North Dakota State University, Fargo, ND 58105.

E-mail address: Friedrich.Littmann@ndsu.edu, Mark.Spanier@ndsu.edu 